\title{
Recognition
}

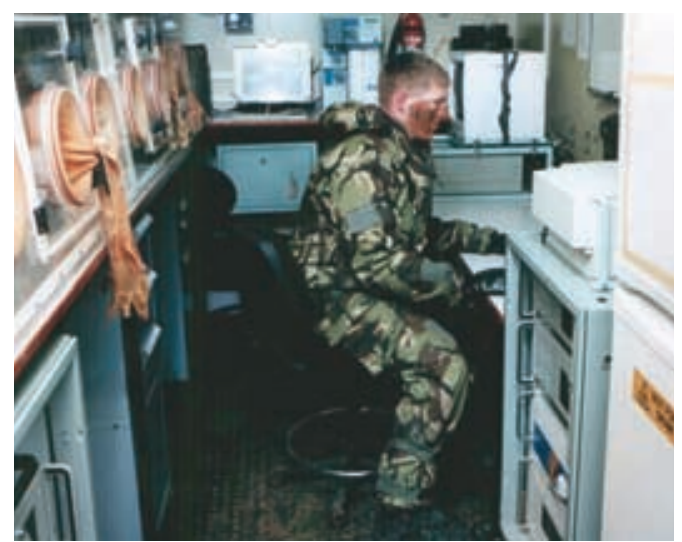

Fig 1. Biological agent detection in the field.

\section{Introduction Overview}

With current technology, it is likely that a BW attack will be completed before the local commander, or his or her medical advisor, is aware that it has taken place. The medical officer must attempt to distinguish between an epidemic of natural origin and a BW attack. Specific considerations include:

- BA are likely to be delivered covertly.

- Sick individuals may be the initial indication that an attack has occurred. Distinguishing a BW attack from background endemic disease may be difficult under some circumstances. Mixed infections, more than one $\mathrm{BW}$ agent used concurrently, or intoxications may occur thereby complicating or delaying diagnosis.

- A large number of casualties may occur during a short period of time.

- In a given geographic area, both military and civilian casualties will occur; effects on animals may be the first sign of an attack.

- Targets may be large geographical areas or smaller, tactically important, objectives. The size of an area in which casualties occur can help narrow the list of likely agents. For example, certain BA, like toxins, can be used most effectively on smaller targets, while others can be disseminated effectively over extremely large areas (for example, anthrax spores).

- Rapid detection and definitive identification of suspected BW agents are essential for tactical, political and forensic as well as medical purposes.
- In the operational theatre atmospheric conditions are critical to the effective use of BA. In general, the optimal time for use of $\mathrm{BW}$ weapons is during the late night and early morning. It is during these hours that inactivation of $\mathrm{BA}$ aerosols by ultra-violet radiation is minimal. In addition, neutral or inversion conditions are most likely to be present at these times. The phenomenon of atmospheric inversion best allows an agent cloud to travel along the land surface.

- Environmental conditions within buildings can impact the effectiveness of a BA release. Examples include airflow, climate control system and the activity of exposed personnel.

\section{WARNING AND DETECTION}

\section{Intelligence}

Adequate, accurate and timely intelligence is required in order to develop an effective defence against BW.

\section{Detection}

BA aerosol detectors can be configured to detect general hazards or specific agents. The positioning of detectors and their sensitivity critically affect their performance. They will be deployed as a network and may be described as located relative to personnel in two ways:

- Upwind of personnel in order to give prior warning of an attack before exposure occurs.

- Co-located with personnel to confirm that exposure has occurred, and aspirationally to provide a detect-to-warn capability.

It should be noted that a single detector may act in both these ways simultaneously for personnel in different locations.

\section{Epidemiological Monitoring}

After an undetected BA attack the first sign of an event may be the appearance of casualties (military, civilian, human or animal). The earlier that patterns of an emerging outbreak of a disease are recognised the greater is the chance of protecting troops who have not yet developed symptoms. Advanced medical surveillance and epidemiological reporting systems with collation and analysis of data can be used to identify spikes of disease on a real time basis. The background level of naturally occurring $\mathrm{BW}$ diseases is a critical 
information requirement for epidemiological reporting. These systems also play an important part in the subsequent assessment and management of a recognised attack. In this context, rapidly deployable biological medical reconnaissance of unusual outbreaks of diseases in the theatre is important.

\section{Prediction of BW Populations at Risk (PAR) and Follow-on Contingency Requirements}

Medical requirements resulting from attacks with BA may be substantially different from those resulting from conventional, nuclear or chemical combat. In most tactical situations, there would be no indication of the presence of BA. The ability to estimate possible casualties and contingency requirements and treatments would assist in the determination of the magnitude of a BW attack:

- Casualty estimation guides (NATO document: AMedP-8), and computer models based on the guides may be fielded by some nations, integrate current available information (operational scenario, agent type, delivery type, attack intensity, atmospheric conditions) and predict the possible effects of future BA attacks on specific PAR.

- The primary purpose of these estimations and models is to predict the appropriate level of medical management (triage, treatment and supportive care) of the BW casualties and the contingency requirements for medical personnel, medical materiel, stockpiles, patient transport or evacuation capabilities and facilities needed to perform treatment and supportive care.

- Since there are limitless attack variables, the current estimates present a limited number of estimates and provisional guidance for estimating cases not modelled. The Command medical officer may need to amplify or modify estimations to meet emergent requirements, such as may result from combined attack (i.e. biological and conventional).

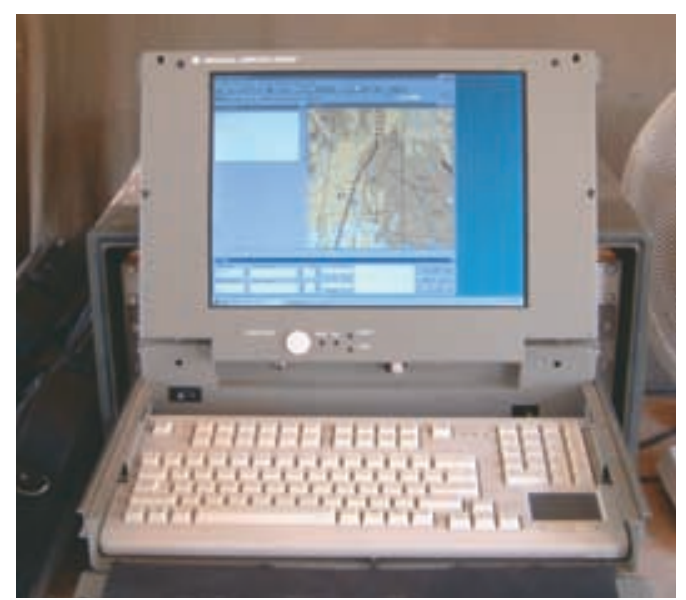

Fig 2. Computerised epidemiology analysis.

\section{EPIDEMIOLOGY}

\section{Difficulties in Detection}

Human beings are a sensitive, and in some cases the only, biodetector. Early clinical findings may be non-specific or atypical of the natural disease. Medical personnel may be unable to differentiate natural disease from BW attacks. Considerable time may elapse following a BW attack before the extent of the exposure is appreciated. Recent advances in aerosol detection enable the rapid identification of some BA. This information would be valuable in the recognition and evaluation of a subsequent outbreak of disease.

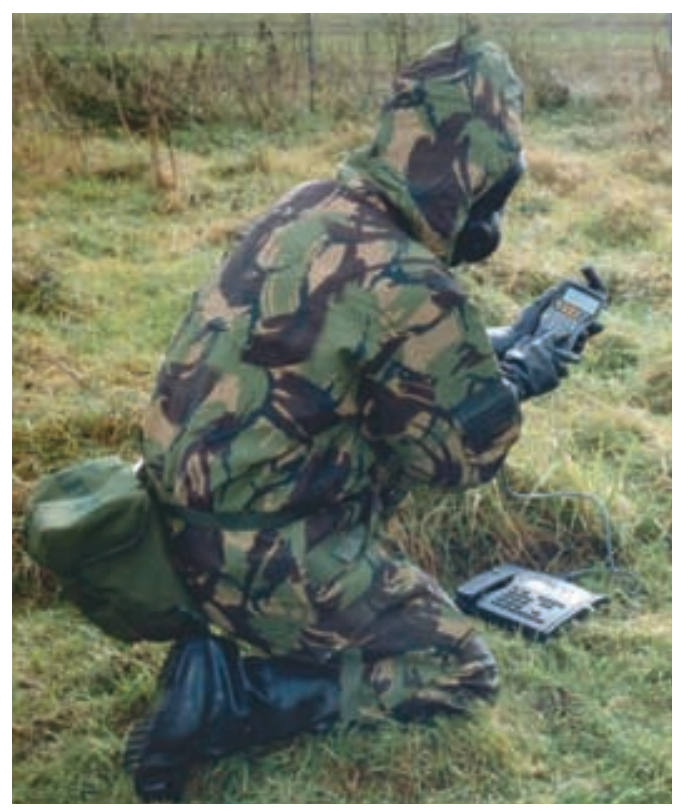

Fig 3. Surveillance Data Entry.

\section{Attack Indicators}

Following a BW attack, the disease pattern may have characteristics that differ from those of a naturally occurring epidemic:

- In contrast to naturally-occurring epidemics (excluding foodborne outbreaks) in which disease incidence increases over a period of weeks or months, the epidemic curve for most large artificially-induced outbreaks is compressed, peaking within a few hours or days (comparable to that during a natural food poisoning outbreak). This is the characteristic pattern of a point source outbreak.

- An understanding of disease epidemiology can be extremely useful in distinguishing natural outbreaks from those induced by BA weapons. For example, diseases that are naturally vector-borne will have environmental parameters that predispose to naturally occurring outbreaks. Appearance of disease in the absence of these parameters would be highly suggestive of a BW attack.

- Defence Medical Services must maintain routine disease surveillance; emergence of 
an atypical pattern mandates immediate notification to a higher authority. The simultaneous appearance of outbreaks in different geographical locations should alert to the possibility of a BA attack. In addition, multiple BA may be used simultaneously in a BW attack, or chemical and biological agents may be combined in a single attack to confuse diagnosis.

\section{Additional Attack Indicators}

Additional indicators of a BW attack include:

- A large number of casualties within the first 48-82 hours after the attack (suggesting an attack with a microorganism), or within hours (suggesting an attack with a toxin). The epidemiology would be that of a massive single source.

- A large number of clinical cases among exposed individuals.

- An illness type highly unusual for the geographic area (for example, Venezuelan equine encephalitis in Europe).

- An illness occurring in an unnatural epidemiological setting, where environmental parameters are not conducive to natural transmission (such as human Venezuelan equine encephalitis in the absence of antecedent disease in horses or in the absence of vector mosquitoes).

- An unusually high prevalence of respiratory involvement in diseases that when acquired in nature, generally cause a non-pulmonary syndrome (the signature of aerosol exposure).

- Casualty distribution aligned with recent wind direction.

- Lower attack rates among those working indoors, especially in areas with filtered air or closed ventilation systems, than in those exposed outdoors. The reverse is true when the attack is made by using ventilation systems in order to disseminate BW indoors.

- Increased numbers of sick or dead animals, often of different species. Some BW agents are capable of infecting/ intoxicating a wide range of hosts.

- Witness to an attack, or discovery of an appropriate delivery system, for example finding a contaminated bomblet or rocket, from which a BA is subsequently isolated and identified.

- Large numbers of rapidly fatal cases, with few recognisable signs and symptoms, resulting from exposure to multiple lethal doses near the dissemination source.

- Unusual entomological parameters.

\section{SAMPLE COLLECTION}

\section{Diagnosis}

The accurate reporting of clinical findings may be critical in alerting other units to both the possibility and nature of a BW attack. Unfortunately, attempts to reach a firm diagnosis on clinical grounds alone may not be productive. However, rapid isolation and differential identification of the BA is of prime importance for patients and risk management. Emerging technology will likely provide provisional diagnostic capabilities locally. However, establishing a definitive diagnosis will often require specialised laboratory facilities.

\section{Environmental Sampling}

General policies for collecting samples in order to facilitate identification of BA are essential. Medical responsibilities normally are limited to collection and submission of diagnostic materials from patients. Environmental sampling is an important element in corroborating the occurrence of a BW attack and involves other agencies such as SIBCRA (Sampling and Identification of Biological Chemical and Radiological Agents) teams (AEP-10: NATO Handbook for Sampling and Identification of Biological and Chemical Agents, Vol 1, Procedures and Techniques, Ed 5). Success or failure in providing a timely medical response will depend upon the rapidity and accuracy of the diagnostic effort, together with the passage of timely information from those organisations involved in environmental sampling. Close co-ordination and co-operation between NBC and Medical staffs will be vital to optimise sampling.

\section{Medical Sampling Principles}

When specialised diagnostic systems are available specimens of blood and other body fluids can be taken to allow rapid agent identification. Swabs of contaminated areas such as the nose or throat can also be employed. The sample taken depends on the apparatus deployed. General principles of the collection and processing of medical samples include the following, notwithstanding the risk from the infective disease:

\section{Specimen Collection.}

Human and animal samples may be collected to aid in definitive diagnosis:

- Blood culture with routine media will readily detect many bacterial agents, although specialised media may be required for some. Both aerobic and anaerobic cultures should be obtained routinely. Cultures and impression smears should be taken from involved lymph nodes, sputum, pleural fluid, cerebrospinal fluid (CSF), and spleen when possible. Appropriate swabs should be taken, the minimum being from the upper respiratory tract.

Acute serum (at least $3 \mathrm{ml}$ for suspected infectious agents, and at least $20 \mathrm{ml}$ for suspected intoxications) should be collected as early as possible after onset 
of symptoms. Blood samples also should be obtained from exposed persons who are not yet symptomatic. Convalescent sera from survivors and non-affected unit members should be obtained 3-4 weeks later.

- Samples for isolation of suspected viral agents should be obtained from organs and tissues as described above, and placed in specialised transport media and frozen for shipment to specified reference laboratories.

- Tissue samples obtained at autopsy should be collected in multiple aliquots: minimally, one (25-50 grams) to freeze for microbial or toxin analysis and one in formalin for histopathology should be obtained. Where possible additional specimens for specialised procedures such as immunofluorescence or polymerase chain reaction studies should be obtained. Organs sampled should include lung, mediastinal lymph nodes, spleen and liver. Obvious lesions and adjacent normal tissue should be taken from affected areas in any organ. Postmortem blood (up to $20 \mathrm{ml}$ ) should be obtained. Consideration should be given to transporting the cadaver in suitable containment to a specialist forensic laboratory.

\section{Specimen Labelling}

- Each container should be labelled with name, numerical identities, type of specimen, and date of collection. Included should be a brief description of the illness and gross autopsy findings; place, date, and time of death; place, date, and time of collection; pathologists; and unit.

- All serum samples should be completely labelled with patient's name, numerical identifier, unit, date, originating medical facility, and medical facility to receive results (if different from submitting facility). Routine laboratory slips should be included with each sample. Data on laboratory slips should include number of days since onset of symptoms, the reason that samples were obtained and a geographical location of collection.

- Clinical and operational data should be included for all samples, together with a form to establish chain of custody. This requirement must be strongly and clearly delineated since evidence may well be politically or militarily disputed.

\section{Specimen Handling and Shipment}

- All specimens from suspected BW casualties should be submitted through the routine diagnostic laboratory chain for processing. Samples must be clearly marked for special diagnostic testing, and chain-of-custody procedures maintained. Samples from biological environments or suspected BW cases may contain dangerous pathogens. Therefore, handling of such specimens should be in agreement with national bio-safety regulations.

- Serum should be contained in appropriate vials that are securely sealed. If possible, each serum sample should be individually placed in a second plastic vial or zip-top bag to prevent leakage. All specimens should be contained in a metal shipping can or other secondary container. Sufficient absorbent material should be packed to prevent leakage outside the container. Samples for microbial or toxin analysis should be kept refrigerated $\left(4-8^{\circ} \mathrm{C}\right)$. If analysis is not to take place within 72 hours samples should be frozen. Formalinfixed material must not be frozen.

- It is the responsibility of the laboratory officer, in concert with the physician, to ensure that suspect specimens are submitted correctly and expeditiously to an appropriate diagnostic laboratory. Shipping must be completed in accordance with both international and national regulations on the transportation of samples.

Forensic Samples and Chain of Evidence

- In the case of SIBCRA the issue of informed consent must be addressed according to existing medical ethical regulations; a special case is the consent for deceased victims. The need to satisfy international law and prove chain of evidence will be paramount after a BW attack.

There may be a requirement to retain a second sample and the following information must be provided:

- When was the sample collected?

- Who maintained custody of the sample?

- What was done with the sample at each change of custody?

\section{IDENTIFICATION OF SPECIFIC BW AGENTS}

\section{Identification Methods}

Methods of identification of BW agents include:

- Isolation of the aetiological agent by culture (possible in one to two days for some BA).

- Detection of toxin by animal inoculation or in-vitro methods.

- Antibody detection (specific immunoglobulin $M(\operatorname{IgM})$ may appear within 3 days).

- Antigen detection via enzyme immunoassay or other sensitive assay methods, eg. immunofluorescence.

- Genome detection employing DNA probes or Polymerase Chain Reaction (PCR).

- Detection of metabolic or degradation 
products of the infectious agent or toxin NATO Laboratory Response Network is in clinical specimens.

- (Electron) Microscopy.

being developed in order to identify the closest laboratory geographically that would meet this requirement. Prior to this

Accredited Laboratories

becoming available, it is suggested that

SIBCRA requires that samples be identified individual countries be approached directly at accredited laboratories for the individual as required for access to accredited BA using an accredited testing method. A laboratories. 